\title{
Seismic tomography using parameter-free Backus-Gilbert inversion
}

\author{
Christophe Zaroli \\ Institut de Physique du Globe de Strasbourg, UMR 7516, Université de Strasbourg, EOST/CNRS, France. \\ E-mail: c.zaroli@unistra.fr
}

\section{SUMMARY}

This proof-of-concept study presents a parameter-free, linear Backus-Gilbert inversion scheme, tractable for seismic tomography problems. It leads to efficient computations of unbiased tomographic images, accompanied by meaningful resolution and uncertainty informations. Moreover, as there is no need to parameterize the model space in this parameter-free approach, it enables numerically accurate data sensitivity kernels to be effectively exploited in tomographic inversions. This is a major benefit over discrete tomographic methods, for which data sensitivity kernels are often inaccurate, as they are projected on a given model parameterization prior to be exploited in the inversion, and these parameterizations are usually coarse to limit the number of parameters and keep tractable the problems of model estimation and/or appraisal. Therefore, this new tomographic scheme fuels great hopes on better constraining multi-scale seismic heterogeneities in the Earth's interior by exploiting accurate data sensitivity kernels, i.e., taking full advantage of known wave-propagation physics, and enabling quantitative appraisals of tomographic features. As a remark, since its computational cost grows as a function of the total number of data squared, it may be better suited to handle moderate-size data sets, typically encountered in regional-scale tomography. Theoretical developments are illustrated within a finite-frequency physical framework. A set of 27070 teleseismic $S$ - 
wave time residuals is inverted, with focus on imaging and appraising shear-wave velocity anomalies lying in the mantle below Southeast Asia, in the $350-1410 \mathrm{~km}$ depth range.

Key words: Inverse theory - Tomography - Body waves

\section{INTRODUCTION}

Tomographic images inferred from seismic data can be exploited to provide constraints within which to frame and answer fundamental questions on the Earth's present-day internal structure, composition and dynamics (e.g., Kennett \& Bunge 2008; Nolet 2008; Romanowicz 2008). Recently, in an effort to build higher resolution tomographic models, finite-frequency effects (e.g., wavefront-healing) present in seismic data have started to be accounted for through the use of finite-frequency data sensitivity kernels (e.g., Dahlen et al. 2000; Hung et al. 2001; Montelli et al. 2004a; Tromp et al. 2005; Mercerat et al. 2014; Zaroli et al. 2015). Interest for finite-frequency tomography has been fueled by continued evidence for structure-related dispersion exhibited in local to global scale sets of $P$ - and $S$-wave cross-correlation time residuals (e.g., Hung et al. 2004; Yang et al. 2006; Sigloch \& Nolet 2006; Zaroli et al. 2010; Hosseini \& Sigloch 2015; Schuberth et al. 2015). However, despite theoretical improvement upon the infinite-frequency approximation of ray theory (e.g., Nolet et al. 2005), several studies have questioned on the actual ability to better constrain small-scale seismic heterogeneities in finitefrequency tomographic models (e.g., Van der Hilst \& de Hoop 2005; Dahlen \& Nolet 2005; Boschi et al. 2006; Chevrot et al. 2012; Maceira et al. 2015; Maguire et al. 2018). Indeed, benefits from using a finite-frequency wave-propagation physical approach may be hampered by several factors, such as the data quality and spatial coverage, and, as further discussed, the inversion scheme.

Most of linear or linearized tomographic inversions to date, within ray-theory or finite-frequency frameworks, have been carried out through two main technical steps (e.g., Aster et al. 2012): 1) Parameterizing the model space with a finite number of parameters; 2) Applying damped least-squares (DLS) methods to estimate these parameters (model estimation), and, though often ignored because of prohibitive computational costs (e.g., Rawlinson et al. 2010, 2014), their resolution and uncertainty (model appraisal). The first step implies that data sensitivity kernels have to be projected on a given parameterization of the model space prior to be effectively exploited in the inversion (e.g., Nolet 2008). Consequently, in order to fully exploit them for modeling finite-frequency effects in the data, it is of crucial importance that finite-frequency data sensitivity kernels remain numerically accurate after such projection. Although there are various ways for parameterizing models (e.g., Sambridge et al. 1995; Montelli et al. 2004b; Ritsema et al. 2011; Chevrot et al. 2012; Zaroli et al. 2015; Maguire et al. 2018), the number of parameters often has to be limited to keep computationally tractable the prob- 
lems of model estimation and/or appraisal. Therefore, finite-frequency sensitivity kernels typically are projected on coarse parameterizations, and thus look like 'fat' ray-theoretical sensitivity kernels (i.e., no sensitivity variation all around the ray path). Those projected sensitivity kernels are then no more suitable for finite-frequency imaging purposes - a major "reason why finite-frequency theory gave so far results similar to ray theory" (Chevrot et al. 2012). Another concern, that links to the second step, is that amplitudes of DLS models may represent locally biased averages over the true-model parameters in regions of poor data illumination (Zaroli et al. 2017). This averaging bias effect is related to adding ad hoc regularization constraints (different from a priori physical constraints) on the model, such as $L_{2}$-norm damping, to remove the non-uniqueness inherent to the least-squares solution (e.g., Menke 1989; Nolet 2008; Voronin \& Zaroli 2018). Since uneven data coverage prevails in local to global scale tomographic experiments, most DLS models are prone to be locally biased - what may lead to model misinterpretations.

In this study, we aim to present a new tomographic scheme that overcomes all the drawbacks related to the previous two technical steps. First of all, a fundamental insight from the pioneering works by Backus \& Gilbert $(1967,1968,1970)$ is that tomographic problems are invariably, at least partly, under-determined: "the collection of Earth models which yield the physically observed values of any independent set of gross Earth data is either empty or infinite dimensional" (Backus \& Gilbert 1967). Recognizing this fact, the linear Backus-Gilbert (B-G) inversion scheme, which belongs to the class of Optimally Localized Averages (OLA) methods, seeks not to construct a particular model solution, i.e., to estimate model parameters, but instead to determine spatially localized, unbiased averages over the continuous true-model properties. Thus, the $\mathrm{B}-\mathrm{G}$ approach seems relevant to move toward parameterfree and unbiased tomography, while enabling to solve all at once the problems of model estimation and appraisal. However, many authors subsequently found it to be too computationally intensive, as well as impractical in the presence of data errors (e.g., Menke 1989; Parker 1994; Trampert 1998; Aster et al. 2012). Recently, following the discrete B-G framework suggested by Nolet (1985), for which a local parameterization of the model space is assumed, Zaroli (2016) uncovered an efficient way of adapting a variant of B-G, namely the SOLA method (Subtractive OLA, proposed by Pijpers \& Thompson (1992) for helio-seismic inversions), to large-scale, linear and discrete seismic tomography problems, even in the presence of data errors. The reader is referred to Zaroli et al. (2017) for a formal comparison of the discrete SOLA and DLS inversion schemes in terms of model estimation and appraisal, as well as for a quantitative illustration of averaging bias effects that may occur in DLS models - both based on synthetic tomographic experiments.

The goal of this study is then to extend the method of discrete SOLA tomography to the parameterfree case, so that the model space can retain its infinite dimensional nature and a specific model 
parametrization never be introduced - and accurate data sensitivity kernels be effectively exploited in the inversion. Sect. 2 presents theoretical developments on this new method, named parameter-free SOLA tomography, within a finite-frequency physical framework (Dahlen et al. 2000). For illustrative purposes, the parameter-free SOLA approach is applied to a finite-frequency inversion of 27070 teleseismic $S$-wave time residuals, with focus on imaging and appraising shear-wave velocity anomalies lying in the mantle below Southeast Asia, in the 350-1410 km depth range. In Sect. 3, parameter-free SOLA tomography is discussed against discrete SOLA and DLS, and several perspectives and future applications are highlighted. Computational aspects involved in parameter-free SOLA tomography are discussed in App. A, including how to reduce the costs and memory requirements.

Finally, this proof-of-concept paper aims to open a new way for solving linear tomographic problems, that: 1) allows accurate data sensitivity kernels to be effectively exploited in tomographic inversions; 2) leads to efficient, embarrassingly parallel, computations of unbiased tomographic images accompanied by meaningful resolution and uncertainty informations, enabling quantitative appraisals of tomographic features; 3 ) is tractable even with limited computational resources, provided moderatesize data sets - as frequently encountered in regional-scale experiments.

\section{PARAMETER-FREE SOLA TOMOGRAPHY}

\subsection{Preamble}

We are interested in linear tomographic problems of the form:

$d_{i}=\int K_{i}(\mathbf{r}) m(\mathbf{r}) d^{3} \mathbf{r}+n_{i}, \quad 1 \leq i \leq N$,

where $d_{i}$ is the $\mathrm{i}$-th datum, $K_{i}$ the sensitivity kernel, $n_{i}$ the noise, and $m$ the 'true' model. As a leitmotiv, we consider the case of finite-frequency $S$-wave time-residual tomography, aimed at imaging three-dimensional shear-wave velocity anomalies in the Earth's mantle. Thus, $m(\mathbf{r})$ denotes the shearwave velocity perturbation in $\mathbf{r}$ with respect to a radial velocity model, $d_{i}$ represents an $S$-wave time residual measured by cross-correlating a pair of observed and synthetic waveforms filtered around a given central period, and $K_{i}(\mathbf{r})$ is a volumetric, $S$-wave time-residual sensitivity kernel which depends on the filtering period (Marquering et al. 1998; Dahlen et al. 2000). In this study, we assume that the noise $\left(n_{i}\right)_{1 \leq i \leq N}$ has zero mean and the data covariance matrix is diagonal: $\mathbf{C}_{d}=\operatorname{diag}\left(\sigma_{d_{i}}^{2}\right)_{1 \leq i \leq N}$. From hereon, both the data $d_{i}$ and sensitivity kernels $K_{i}$ are scaled by the data errors $\sigma_{d_{i}}$.

In our view, discretizing a finite-frequency sensitivity kernel $K_{i}$ on a grid made of $50 \mathrm{~km}$ edgelength cubic cells is sufficient to fully capture its form (i.e., all its characterizing sensitivity variations), as illustrated in Fig. 1(a) for an SS phase. Discretizing sensitivity kernels is necessary to perform nu- 
merical calculations, but is fundamentally different from discretizing the model space. That is, discretizing the model space leads sensitivity kernels to be projected onto the model parameterization prior to be exploited in the inversion. Fig. 1(b) shows the projection of the same SS sensitivity kernel on a tomographic grid made of $200 \mathrm{~km}$ edge-length cubic cells. One sees that the projected kernel looks like a 'fat' ray-theoretical sensitivity kernel, i.e., there is almost no sensitivity variation all around the ray path. Such a projected kernel is no more suitable for finite-frequency imaging purposes.

Most tomographic experiments rely on worldwide distributed earthquakes and/or receivers, for example see Fig. 2(a), so that sensitivity kernels may sample any part of the mantle, and, therefore, the entire mantle needs to be parameterized. To fully capture the form of every finite-frequency sensitivity kernels, one could parameterise the whole mantle using $50 \mathrm{~km}$ edge-length cubic cells, which would lead to 7.2 millions parameters and then make intractable the problems of model estimation and/or appraisal.

In the following, we aim at showing from theory to practice that parameter-free SOLA tomography: 1) provides an efficient way to circumvent the need for parameterizing the model space, enabling numerically accurate sensitivity kernels to be exploited in tomographic inversions; 2) leads to efficient, all-at-once, computations of unbiased tomographic images, accompanied by resolution and uncertainty informations.

\subsection{Theory}

In the B-G approach, one explicitly seeks an estimate, $\hat{m}^{(k)}$, that represents a weighted average over the continuous true-model properties, $m(\mathbf{r})$. This averaging process takes place through an averaging kernel, $A^{(k)}(\mathbf{r})$, that we wish to be spatially localized around a given query point, $\mathbf{r}^{(k)}$. This leads to writing:

$\hat{m}^{(k)}=\int A^{(k)}(\mathbf{r}) m(\mathbf{r}) d^{3} \mathbf{r} \quad(+$ propagated noise $)$.

We wish that the integral $\int A^{(k)} m$ yields unbiased averages over the true model $m$. The averaging kernel $A^{(k)}$ should then satisfy to the following 'unimodular condition':

$\int A^{(k)}(\mathbf{r}) d^{3} \mathbf{r}=1$

and also be non-negative. As a remark, the model estimate $\hat{m}^{(k)}$ is said to be biased (Nolet 2008) if the averaging kernel $A^{(k)}$ does not meet (3). Zaroli et al. (2017) show (though in a discrete framework) that if $\int A^{(k)}$ is larger (smaller) than one, then $\hat{m}^{(k)}$ may be biased toward higher (lower) amplitude values, respectively, and thus not represent anymore a true averaging over the true model. They demonstrate that this averaging bias effect may occur in DLS models, especially in regions with poor data 


\section{6}

Christophe Zaroli

coverage. Since B-G (or SOLA) estimates are explicitly constrained to meet (3), they are expected to be unbiased. Averaging kernels, also referred to as resolving kernels, inform us on the local resolving length in tomographic images, i.e., the minimum size of velocity anomalies that could be locally detected. For example, if a resolving kernel $A^{(k)}$ was constant-valued inside a 3-D ball centred on a query point $\mathbf{r}^{(k)}$ and zero elsewhere, then the ball's radius would correspond to the resolving length that one could, at best, expect to reach in $\mathbf{r}^{(k)}$. Since the forward problem (1) is linear, one can seek the estimate $\hat{m}^{(k)}$ as a linear combination of the data:

$\hat{m}^{(k)}=\sum_{i=1}^{N} x_{i}^{(k)} d_{i}$,

where the $N$ unknown, real-valued coefficients

$\mathbf{x}^{(k)}=\left(x_{i}^{(k)}\right)_{1 \leq i \leq N}$

represent a generalized inverse operator that maps the data to the estimate. From (1), (2) and (4), one can write the estimate as:

$\hat{m}^{(k)}=\int \underbrace{\left(\sum_{i=1}^{N} x_{i}^{(k)} K_{i}(\mathbf{r})\right)}_{A^{(k)}} m(\mathbf{r}) d^{3} \mathbf{r}+\underbrace{\sum_{i=1}^{N} x_{i}^{(k)} n_{i}}_{\begin{array}{c}\text { propagated } \\ \text { noise }\end{array}}$,

and the resolving kernel $A^{(k)}$ can be formally expressed as a linear combination of the sensitivity kernels:

$A^{(k)}(\mathbf{r})=\sum_{i=1}^{N} x_{i}^{(k)} K_{i}(\mathbf{r})$.

The term $\sum_{i} x_{i}^{(k)} n_{i}$ in (6) represents the amount of data noise that propagates into $\hat{m}^{(k)}$. The variance in the model estimate $\hat{m}^{(k)}$ can be expressed as:

$\sigma_{\hat{m}^{(k)}}^{2}=\sum_{i=1}^{N}\left(x_{i}^{(k)}\right)^{2}\left(\sigma_{d_{i}}\right)^{2}=\sum_{i=1}^{N}\left(x_{i}^{(k)}\right)^{2}$,

since the data were scaled by their errors. The uncertainty $\sigma_{\hat{m}^{(k)}}$ informs us on the level of propagated noise in the 'weighted average' estimate $\hat{m}^{(k)}$. As a remark, $\sigma_{\hat{m}^{(k)}}$ cannot inform us on how much $\hat{m}^{(k)}$ may differ from the true-model value $m\left(\mathbf{r}^{(k)}\right)$ - at least when the spatial variations of $m$ are non smooth and/or the spatial extent of $A^{(k)}$ is far from a Dirac delta function. Both the resolving kernels and uncertainties are needed for quantitative model appraisals, to apprehend whether emerging structures in tomographic images are resolved given the data and their errors (see Sect. 2.4). Once the generalized inverse $\mathbf{x}^{(k)}$ is known, one can directly infer the estimate $\hat{m}^{(k)}$, resolving kernel $A^{(k)}$ and 
uncertainty $\sigma_{\hat{m}^{(k)}}$ :

$\mathbf{x}^{(k)} \Longrightarrow\left\{\begin{array}{lll}\sum_{i=1}^{N} x_{i}^{(k)} d_{i} & \longrightarrow \hat{m}^{(k)} \\ \sum_{i=1}^{N} x_{i}^{(k)} K_{i}(\mathbf{r}) & \longrightarrow A^{(k)}(\mathbf{r}) \\ \left(\sum_{i=1}^{N}\left(x_{i}^{(k)}\right)^{2}\right)^{1 / 2} & \longrightarrow \sigma_{\hat{m}^{(k)}}\end{array}\right.$

The B-G approach consists in directly solving for the generalized inverse $\mathbf{x}^{(k)}$, such that $\mathbf{x}^{(k)}$ leads to the most peak-shaped resolving kernel $A^{(k)}$ around the query point $\mathbf{r}^{(k)}$, while moderating at most the propagated noise, i.e., minimizing the variance $\sigma_{\hat{m}^{(k)}}^{2}$. Zaroli (2016) has introduced and adapted to large-scale, linear and discrete seismic tomography problems the SOLA method, an alternative B-G formulation which retains all its advantages but is more efficient and versatile in the explicit construction of resolving kernels (Pijpers \& Thompson 1992). We aim at extending the discrete SOLA tomographic method to the parameter-free case, named parameter-free SOLA tomography. The key idea of SOLA is to specify an a priori target form $T^{(k)}$ for each resolving kernel $A^{(k)}$. Those 'target' resolving kernels, $T^{(k)}$, are referred as target kernels for short. In the parameter-free case, a target kernel $T^{(k)}$ is formally defined as follows:

$T^{(k)}(\mathbf{r})= \begin{cases}1 / \int_{\mathbf{r} \in \mathbb{S}^{(k)}} d^{3} \mathbf{r} & \text { if } \mathbf{r} \in \mathbb{S}^{(k)} \\ 0 & \text { elsewhere }\end{cases}$

where $\mathbb{S}^{(k)}$ is a volumetric region well localized in the model space (e.g., a ball or a spheroid), which is centred on $\mathbf{r}^{(k)}$ and whose size represents an a priori estimate of the local resolution. Note that (10) implies that target kernels also satisfy to the unimodular condition:

$\int T^{(k)}(\mathbf{r}) d^{3} \mathbf{r}=1$

Rather than minimizing the spread of each resolving kernel, SOLA aims at minimizing the integrated squared difference between each resolving kernel and its associated target kernel. That is, for every query point, $\mathbf{r}^{(k)}$, the parameter-free SOLA minimization problem consists in finding the coefficients $\mathbf{x}^{(k)} \in \mathbb{R}^{N}$ such that:

$$
\left\{\begin{aligned}
& \underbrace{\int\left[A^{(k)}(\mathbf{r})-T^{(k)}(\mathbf{r})\right]^{2} d^{3} \mathbf{r}}_{\begin{array}{c}
\text { resolution } \\
\text { misfit }
\end{array}}+\eta^{2} \underbrace{\sigma_{\hat{m}(k)}^{2}}_{\substack{\text { model } \\
\text { variance }}}=\min \\
\text { s.t. } & \underbrace{\int A^{(k)}(\mathbf{r}) d^{3} \mathbf{r}=1}_{\begin{array}{c}
\text { unimodular } \\
\text { condition }
\end{array}} .
\end{aligned}\right.
$$

Since the value of the trade-off (resolution $v s$ uncertainty) parameter $\eta$ is free to differ for every query point $\mathbf{r}^{(k)}$, one should rather write it as $\eta^{(k)}$. In this study, we choose it to be constant-valued and then drop the $k$ subscript. Indeed, as suggested by Zaroli (2016) and Zaroli et al. (2017), a constant trade- 


\section{8}

Christophe Zaroli

off parameter $\eta$ may lead to "globally coherent" tomographic images when the size of target kernels is set to spatially vary as ray density, that is, a proxy for the a priori local resolution (see Sect. 2.4). The parameter-free SOLA minimization problem (12) can be written in the matrix form:

$\left\{\begin{aligned} & \mathbf{F}^{(\eta)} \mathbf{x}^{(k)}=\mathbf{u}^{(k)} \\ \text { s.t. } & \mathbf{c}^{\mathrm{T}} \mathbf{x}^{(k)}=1,\end{aligned}\right.$

where elements of the symmetric matrix $\mathbf{F}^{(\eta)}=\left(F_{i i^{\prime}}^{(\eta)}\right)_{1 \leq i, i^{\prime} \leq N}$, and vectors $\mathbf{c}=\left(c_{i}\right)_{1 \leq i \leq N}$ and $\mathbf{u}^{(k)}=\left(u_{i}^{(k)}\right)_{1 \leq i \leq N}$ are given by:

$\left\{\begin{aligned} F_{i i^{\prime}}^{(\eta)} & =\int K_{i}(\mathbf{r}) K_{i^{\prime}}(\mathbf{r}) d^{3} \mathbf{r}+\eta^{2} \delta_{i i^{\prime}} \\ c_{i} & =\int K_{i}(\mathbf{r}) d^{3} \mathbf{r} \\ u_{i}^{(k)} & =\int T^{(k)}(\mathbf{r}) K_{i}(\mathbf{r}) d^{3} \mathbf{r},\end{aligned}\right.$

with $\delta$ the Kronecker symbol. Though systems as (13) are usually solved using Lagrange multipliers, we rather follow Nolet (1985) and Zaroli (2016) and use an LSQR-based approach. Let us consider the three column-vectors (assuming $c_{1} \neq 0$ ):

$\hat{\mathbf{x}}^{(k)}=\left(x_{i}^{(k)}\right)_{2 \leq i \leq N}, \quad \hat{\mathbf{c}}=\left(\frac{c_{i}}{c_{1}}\right)_{2 \leq i \leq N}, \quad \mathbf{e}_{1}=\left(\delta_{i 1}\right)_{1 \leq i \leq N}$.

We wish the resolving kernel $A^{(k)}$ to satisfy to the unimodular condition (3), which may also be written as follows:

$\mathbf{c}^{\mathrm{T}} \mathbf{x}^{(k)}=1$

The first element of $\mathbf{x}^{(k)}$ can be expressed in terms of the others:

$x_{1}^{(k)}=c_{1}^{-1}-\hat{\mathbf{c}}^{\mathrm{T}} \hat{\mathbf{x}}^{(k)}$,

and the vector $\mathbf{x}^{(k)}$ be written in function of $\hat{\mathbf{x}}^{(k)}$, that is:

$\mathbf{x}^{(k)}=\mathbf{B} \hat{\mathbf{x}}^{(k)}+c_{1}^{-1} \mathbf{e}_{1}$,

where the matrix $\mathbf{B}$ is defined as:

$\mathbf{B}=\left(\begin{array}{c}-\hat{\mathbf{c}}^{\mathrm{T}} \\ \mathbf{I}_{N-1}\end{array}\right)$,

with $\mathbf{I}_{N-1}$ the identity matrix of order $N-1$. The parameter-free SOLA problem (13) consists in solving for $\hat{\mathbf{x}}^{(k)}$ the system:

$\mathbf{H}^{(\eta)} \hat{\mathbf{x}}^{(k)}=\mathbf{v}^{(k, \eta)}$, 
and then inferring the generalized inverse solution $\mathbf{x}^{(k)}$ from $\hat{\mathbf{x}}^{(k)}$, where the matrix $\mathbf{H}^{(\eta)}$ and vector $\mathbf{v}^{(k, \eta)}$ are defined as:

$$
\left\{\begin{array}{l}
\mathbf{H}^{(\eta)}=\mathbf{F}^{(\eta)} \mathbf{B} \\
\mathbf{v}^{(k, \eta)}=\mathbf{u}^{(k)}-\mathbf{F}^{(\eta)} c_{1}^{-1} \mathbf{e}_{1} .
\end{array}\right.
$$

We use LSQR (Paige \& Saunders 1982) to numerically solve (20); for a given $\eta$, it iteratively converges to the solution:

$$
\hat{\mathbf{x}}^{(k, \eta)}=\underset{\hat{\mathbf{x}}^{(k)} \in \mathbb{R}^{N-1}}{\arg \min }:\left\|\mathbf{v}^{(k, \eta)}-\mathbf{H}^{(\eta)} \hat{\mathbf{x}}^{(k)}\right\|^{2},
$$

where $\|\cdot\|$ denotes the $L_{2}$-norm.

\subsection{Numerical considerations}

Since a constant-valued trade-off parameter $\eta$ is assumed, there are $P$ parameter-free SOLA tomographic systems (20) to be set up, and solved, where $P$ is the total number of query points $\mathbf{r}^{(k)}$. Firstly, one needs to compute $P$ vectors $\mathbf{v}^{(k, \eta)}=\left(v_{i}^{(k, \eta)}\right)_{1 \leq i \leq N}$, with:

$v_{i}^{(k, \eta)}=\underbrace{\int T^{(k)} K_{i}}_{u_{i}^{(k)}}-\underbrace{\left[\int K_{i} K_{1}+\eta^{2} \delta_{i 1}\right]}_{F_{i 1}^{(\eta)}} c_{1}^{-1}$.

This task mainly consists in calculating, at most, $P \times N$ integrals $\int T^{(k)} K_{i}$; it is cheap to compute the small fraction of non-zero integrals $\int K_{i} K_{1}$ (see App. A5). In this study, we assume that there are much more data than query points, i.e., $P / N \ll 1$ (for example, $P / N \simeq 16 \%$ in Sect. 2.4). Our view is that there is no need to consider too many query points, that is, not more than required to fit the spatial variations of the a priori local resolution; the same argument holds for data-driven, irregular tomographic grids (e.g., Sambridge et al. 1995; Nolet \& Montelli 2005; Zaroli et al. 2015). Concerning the application in Sect. 2.4, we report that it is not costly to compute, in parallel, all those, at most, $P \times N \ll N^{2}$ integrals $\int T^{(k)} K_{i}$. Numerical details for calculating them are discussed in App. A. Secondly, one has to compute the matrix $\mathbf{H}^{(\eta)}$, of size $N \times(N-1)$, whose elements are given by:

$H_{\mu \nu}^{(\eta)}=\underbrace{\int K_{\mu} K_{\nu+1}+\eta^{2} \delta_{\mu, \nu+1}}_{F_{\mu, \nu+1}^{(\eta)}}-\underbrace{\left[\int K_{\mu} K_{1}+\eta^{2} \delta_{\mu 1}\right]}_{F_{\mu 1}^{(\eta)}} \underbrace{\frac{c_{\nu+1}}{c_{1}}}_{\hat{c}_{\nu}}$,

where $1 \leq \mu \leq N$ and $1 \leq \nu \leq N-1$. Since the matrix $\mathbf{H}^{(\eta)}$ does not depend on the query point, it does not need to be recomputed $P$ times. This nice property is due to the SOLA formulation itself - a crucial advantage compared to the B-G approach (e.g., Pijpers 1997). The matrix $\mathbf{H}^{(\eta)}$ can be easily derived from the symmetric matrix $\mathbf{F}^{(\eta)}$ of order $N$. The main computational difficulty of parameterfree SOLA tomography, compared to discrete SOLA, arises from the calculation of $\mathbf{F}^{(\eta)}$. Though it is 
not costly to compute its $N$ diagonal elements, $F_{i i}^{(\eta)}=\int K_{i}^{2}+\eta^{2}$, it may be expensive to compute its $N(N-1) / 2$ off-diagonal elements, $F_{i i^{\prime}}^{(\eta)}=\int K_{i} K_{i^{\prime}}$. Indeed, as the number of integrals $\int K_{i} K_{i^{\prime}}$ grows as a function of $N^{2}$, this may be a computational burden when facing large data sets (see Sect. 3), especially if one aims at fully capturing the form of finite-frequency sensitivity kernels (see Sect. 2.1). Numerical details for calculating $\mathbf{F}^{(\eta)}$ are given in App. A. It is straightforward to calculate $\mathbf{H}^{(\eta)}$ and $\mathbf{v}^{(k, \eta)}$ for different $\eta$ values. Last, but not least, it is worth of noting that parameter-free SOLA tomography is well suited for parallel computations, since the problem can be easily separated into a number of independent computational tasks, for example to calculate all the $P$ vectors $\mathbf{v}^{(k, \eta)}$, the $\sim N^{2} / 2$ integrals $\int K_{i} K_{i^{\prime}}$, and the $P$ LSQR-solution vectors $\hat{\mathbf{x}}^{(k, \eta)}$. Finally, we show in App. A how to reduce the computational costs and memory requirements involved in setting up and solving parameter-free SOLA tomographic systems, tailored to teleseismic finite-frequency body-wave mantle tomography.

\subsection{Application}

We aim at applying the parameter-free SOLA tomographic method, within a finite-frequency physical framework (Dahlen et al. 2000), to the problem of imaging and appraising isotropic variations of shear-wave velocities in the mantle region below Southeast Asia, with respect to the reference radial velocity model iasp91 (Kennett \& Engdahl 1991). The Southeast-Asia region, depicted in Fig. 2(b), is chosen without any particular purpose, except that it is characterized by several deep subduction systems, and because of a large amount of data available in that region, making it worth for first time testing parameter-free SOLA tomography.

Our data set consists in $N=27070$ teleseismic $S$ and $S S$ time residuals, measured by crosscorrelation technique at $22 \mathrm{~s}$ central period (passband Gaussian filter) (Zaroli et al. 2010). The associated, globally distributed earthquakes and receivers are shown in Fig. 2(a). These data are a subset of those used in previous, global-scale, discrete SOLA and DLS tomographic experiments (Zaroli 2016). To generate it, a selection criterion was applied to ensure that every data sensitivity kernels sample the mantle region of interest. Estimates of data errors include earthquake-location and measurementprocess errors (Zaroli et al. 2010, 2013); these original errors were additionally increased by 30 per cent by Zaroli (2016) to have unit reduced chi-square for a global DLS model. Each finite-frequency sensitivity kernel is calculated at $22 \mathrm{~s}$ period on a grid made of $50 \mathrm{~km}$ edge-length cubic cells (see Fig. 1(a) and App. A1), using analytical formulas (Zaroli et al. 2013) for which is assumed a Gaussian source power spectrum (Hung et al. 2001).

Each target kernel $T^{(k)}$ is a spheroid centred on a query point. We follow Zaroli (2016) to specify the locations and sizes of target kernels. We use the ray density as a first-order proxy for the spatial 
variations of the a priori local resolution, make an educated guess about the a priori resolving-length bounds, and then interpolate to determine the sizes of target kernels at given locations. To limit the number of query points, their locations are chosen such that they fit the spatial distribution of the $a$ priori local resolving length. Fig. 3 displays lateral views of all the $P=4310$ target kernels. They are spanning the region of interest at eight depths in the $350-1410 \mathrm{~km}$ range, where our data coverage is the most relevant. The lateral radius of target kernels is driven by ray density; it ranges from 200 to $1000 \mathrm{~km}$ and represents the $a$ priori, isotropic, local, lateral resolving length. Their radial radius gradually varies from 130 to $200 \mathrm{~km}$ in the $350-1410 \mathrm{~km}$ depth range, respectively, and represents the a priori, local, radial resolving length. A constant value is chosen for the trade-off parameter $\eta$, after having tested a few different values. Note that SOLA solutions depend on tunable inversion parameters (target kernels and trade-off parameter), so that different choices would result in different, unbiased model estimates and appraisals - leading to different, fully quantitative and thus meaningful (at least in a mathematical sense) model interpretations. As a remark, in the case of a synthetic tomographic problem, Zaroli et al. (2017) illustrate the variability of SOLA solutions (i.e., model, resolution, uncertainty) as a function of the trade-off parameter $\eta$.

Conventional tomographic images can be built, for plotting purposes, from linear interpolations among the model estimates, $\hat{m}^{(k)}$. Fig. 4 shows the resulting images in the upper mantle $(350 \mathrm{~km}$ depth), transition zone $(465,595 \mathrm{~km}$ depth) and mid-lower mantle $(735,885,1035,1210,1410 \mathrm{~km}$ depth). Some features seem to be worth of further investigations. For example those characterized by strongly positive velocity anomalies (bluish); some of them correspond to major deep subducted slabs, as in the Sumatra and Java regions (indicated in Fig. 2(b)). Even more interesting are the negative anomalies (reddish) appearing on West-Southwest side of the Sumatra slab in the $350-1035 \mathrm{~km}$ depth range (see Figs. 4(a-f)), while none are showing up nearby on South side of the Java slab. Quantitatively interpreting these complex structural features would require to analyse them in the light of resolving kernels and uncertainties, which is beyond the scope of this work (see Sect. 3). As a remark, Zaroli et al. (2017) report that for their synthetic tomographic experiments, the discrete SOLA models do fit the data at the same level as the DLS models - while the SOLA method is not specifically aimed at minimizing the data misfit. In this study, one cannot compute the data misfit because the parameterfree SOLA model is calculated in a limited region (Southeast Asia), while the data coverage spans almost the whole mantle.

Fig. 5 shows interpolated maps of uncertainties, $\sigma_{\hat{m}^{(k)}}$. They merely represent the amount of data noise that propagates into the model estimates, and come with some underlying assumptions on the noise itself (which is assumed to follow uncorrelated, zero-mean, Gaussian statistics). While the amplitudes of the model estimates are within $\pm 3 \%$, one sees that their uncertainties may reach at most 
$0.4 \%$. As expected, the spatial variations of uncertainties are similar to those of the ray-density driven target kernels (see Fig. 3). In the regions where the size of target kernels is large (small), i.e., the $a$ priori local resolution is poor (good), the uncertainty is low (high), respectively - the unavoidable trade-off between resolution and uncertainty (e.g., Menke 1989).

Resolving kernels, $A^{(k)}$, have to be calculated in a consistent way with respect to the discretization of data sensitivity kernels. In this study, each resolving kernel is then computed on a grid which consists in $50 \mathrm{~km}$ edge-length cubic cells surrounding the considered query point (see App. A4), enabling us to fully capture its form. Fig. 6 shows three examples of resolving kernels, associated to three query points located below Sulawesi Island at 350, 595 and $1035 \mathrm{~km}$ depth. Horizontal and vertical crosssections through those resolving kernels are shown. As expected for teleseismic $S$-wave tomography, their lateral (radial) extent is smaller (larger) in the upper than lower mantle, respectively. That is, vertical smearing (horizontal leaking) mainly occurs in the upper (lower) mantle, respectively. As a remark, one possible artifact that SOLA could be prone to arises when resolving kernels are significantly negative, since they cannot be considered as truly averaging kernels. This does not appear to be the case in this study; for example, only weak negative values are observed in Fig. 6. Pijpers \& Thompson (1994) and Zaroli et al. (2017) discuss how to avoid such artifacts by enlarging the size of target kernels. Note that the target kernels may differ from the actual resolving kernels (see Fig. 6). This simply means that the a priori local resolving length was chosen too optimistically. However, as long as the resolving kernels are mostly non-negative and spatially well localized, they can be exploited for appraising the actual local resolution in tomographic images.

Finally, to illustrate the appraisal of tomographic features with the parameter-free SOLA approach, we discuss whether is resolved the lateral flattening of the Sumatra-Java slabs in mid lower mantle. Indeed, the lateral extent of these slabs appears to be much smaller in the upper mantle (see Fig. 4(a)) and transition zone (see Fig. 4(b-c)), compared to mid lower mantle (see Fig. 4(d-g)). To have a clue on the variations with depth of the lateral resolving length in this region, let us consider the three resolving kernels shown in Fig. 6 and estimate their lateral extent, i.e., the local lateral resolving length. We report that, below Sulawesi Island, the lateral resolving length is, at most, $200 \mathrm{~km}(300,500)$ at a depth of $350 \mathrm{~km}(595,1035)$, respectively. In particular, Fig. 6(c) indicates that the lateral extent of these slabs around Sulawesi Island at $1035 \mathrm{~km}$ depth is much larger than the local lateral resolving length. Moreover, model estimates are 0.95, 0.92 and 1.03 percents for the corresponding query points below Sulawesi Island at 350, 595 and $1035 \mathrm{~km}$ depth, while their uncertainties are three times smaller, that is, $0.33,0.30$ and 0.29 , respectively. Thus, one can argue that the slab lateral flattening that takes place in mid lower mantle, at least below Sulawesi Island, is resolved given our data and their errors. 


\section{DISCUSSION AND PERSPECTIVES}

First, we aim to discuss when parameter-free SOLA tomography should be preferred to discrete SOLA tomography, and vice versa. Let $M^{\infty}$ be the minimum number of parameters required to parameterise the entire model space, so that every projected sensitivity kernels are accurate. In the context of teleseismic, finite-frequency, $S$-wave mantle tomography, it leads to $M^{\infty} \simeq 10^{6}-10^{7}$ (see Sect. 2.1). If one aims at fully exploiting finite-frequency theory, but cannot handle discrete SOLA inversions with $M^{\infty}$ parameters, then one should definitely use the parameter-free SOLA approach. However, if the total number of data is too high, for example $N \gg 10^{5}$, it may not be tractable to compute the $\sim N^{2} / 2$ elements of the matrix $\mathbf{F}^{(\eta)}$ (see Sect. 2.3, App. A). Hence, one may have no choice but to move back to discrete SOLA with a total number of parameters $M \ll M^{\infty}$ (and thus simply have to project $N$ sensitivity kernels on a given, coarse tomographic grid). Consequences would be that some, if not all, projected sensitivity kernels would become unsuitable for finite-frequency imaging purposes (see Fig. 1(b)). Note that parameter-free SOLA tomography is particularly well suited for regional-scale experiments, for which moderate-size data sets are typically encountered, enabling to take full advantage of finite-frequency theory even with modest computational resources (see App. A6).

As an additional comparison of parameter-free SOLA versus other tomographic schemes, let us reconsider the standard, discrete DLS approach, and focus on the problem of imaging and appraising deep mantle plumes, a topic of high interest, recently revisited by Maguire et al. (2018). In their study, various plume models and earthquakes-receivers settings are considered to generate synthetic sets of teleseismic body-wave time residuals, inverted using DLS and finite-frequency sensitivity kernels. Relying on powerful computational facilities, they are able to parameterize the entire mantle using a cartesian cubed sphere approach (e.g., Ronchi et al. 1996), which consists in $\sim 3.5$ millions roughly cubic cells ( $\sim 65 \mathrm{~km}$ edge length), enabling projected finite-frequency sensitivity kernels to be accurate. As a remark, other recent studies (e.g., Charléty et al. 2013; Nolet et al. 2019) were able to derive teleseismic, DLS-based tomographic images when using so many parameters. However, handling millions parameters makes prohibitive to compute the full DLS generalized inverse (e.g., Bogiatzis et al. 2016). Hence, resolution and uncertainty informations cannot be fully taken into account to quantitatively analyze, e.g., plume-like, features in DLS images. Note that the typical size of Maguire et al. (2018)'s data sets is $N \simeq 5 \cdot 10^{4}$, what parameter-free SOLA can handle with relatively modest resources and while solving all at once both the imaging and appraising problems. Moreover, DLS images may be locally biased in regions with poor data illumination, due to ad hoc regularization, such as below isolated receivers where ray paths are quasi-vertical (Zaroli et al. 2017) - while SOLA solutions are explicitly constrained to be unbiased. Maguire et al. (2018) identify part of this bias effect. Assuming an hypothetical vertical conduit of 'slow' anomalies in the mantle below Hawaii, 
they show that the recovered plume may contain prominent 'fast' anomalies - what is a reminder that DLS-based tomographic images could lead to physical misinterpretations.

In the following, we aim to highlight some perspectives related to parameter-free SOLA tomography. First, since it yields unbiased images with resolution and uncertainty informations, one could naturally aim at evaluating in a fully quantitative way whether specific features of interest (e.g., mantle plumes, slabs) are resolved, or not. Future work could consist in designing algorithms aimed at better apprehending and visualizing this new wealth of available informations (model, resolution, uncertainty), enabling to quickly identify resolved features. Since reliable estimates of model uncertainties $\sigma_{\hat{m}^{(k)}}$ require reliable estimates of data errors $\sigma_{d_{i}}$, one could aim at better evaluating the noise contributions in various data sets, and also to investigate the impact of assuming a wrong noise model on the SOLA results.

In a similar line to earlier tomographic filtering studies (e.g., Ritsema et al. 2007; Schuberth et al. 2009; Davies et al. 2012; Zaroli et al. 2017), one could investigate how user-defined input features (e.g., mantle plumes, subducted slabs, whole-mantle geodynamical models) are seen through SOLA resolving kernels, i.e., analyzing the term 'filtered input model' in (25). Furthermore, with the explicit knowledge of the SOLA generalized inverse, one could also investigate the amount of data noise which is expected to propagate into the recovered tomographic features. That is, the output model estimate $\hat{m}_{\text {out }}^{(k)}$ for a specific query point can be related to a given input model $m_{\text {in }}$ as follows:

$$
\begin{aligned}
& \hat{m}_{\text {out }}^{(k)}=\sum_{i} x_{i}^{(k)} \underbrace{\left(\int K_{i} m_{\text {in }}+n_{i}^{\text {synth }}\right)}_{\text {synthetic data, } d_{i}^{\text {synth }}} \\
& =\underbrace{\int A^{(k)} m_{\text {in }}}_{\substack{\text { filtered } \\
\text { input model }}}+\underbrace{\sum_{i} x_{i}^{(k)} n_{i}^{\text {synth }}}_{\substack{\text { propagated } \\
\text { synthetic noise }}}
\end{aligned}
$$

where the $\mathrm{i}$-th synthetic noise component $n_{i}^{\text {synth }}$ is randomly drawn from a zero-mean normal distribution with unit standard deviation (since the original tomographic system (1) was scaled by the data errors), and the generalized inverse components $x_{i}^{(k)}$ and the resolving kernel $A^{(k)}$ are those from the actual SOLA tomographic experiment. Therefore, as in the discrete SOLA case (Zaroli et al. 2017), the parameter-free SOLA framework provides an efficient and fully quantitative way for comparing input and output features, by means of analyzing the filtered input model and the propagation of synthetic noise into the tomographic model solution. Note that the parameter-free SOLA 'filtered input model' can also be indirectly computed as: $\int A^{(k)} m_{\text {in }}=\sum_{i} x_{i}^{(k)} \int K_{i} m_{\text {in }}$. This may be useful if it is cheaper to compute the $N$ integrals $\int K_{i} m_{\text {in }}$ compared to the $P$ integrals $\int A^{(k)} m_{\text {in }}$ (requiring all the resolving kernels to be explicitly calculated). 
The parameter-free SOLA approach could also be exploited to invert onset-time data, for which sensitivity kernels are infinitesimally narrow rays. Since one expects less pairs of crossing rays, compared to crossing finite-frequency kernels, it should be cheaper to compute the integrals $\int K_{i}^{\text {ray }} K_{i^{\prime}}^{\text {ray }}$. Onset-time and correlation-time data, modeled by rays and finite-frequency sensitivity kernels (e.g., Montelli et al. 2004b; Obayashi et al. 2013; Hosseini 2016), could also be jointly inverted with SOLA. In addition, since fully accurate data sensitivity kernels are exploited in parameter-free SOLA inversions, one could revisit comparisons of ray-theory $v s$ finite-frequency $v s$ multi-frequency tomography, while comparing differences in terms of the images and their appraisals. Finally, parameter-free SOLA tomography could be applied to other linear problems, such as, for example, finite-frequency surfacewave tomography (e.g., Zhou et al. 2005; Nolet 2008).

\section{CONCLUSION}

We have presented a parameter-free, linear Backus-Gilbert inversion scheme, tractable for seismic tomography problems - named parameter-free SOLA tomography. Theoretical and numerical developments have been illustrated for teleseismic body-wave mantle tomography, in a finite-frequency physical framework. This new tomographic scheme leads to efficient, embarrassingly parallel, computations of unbiased images, accompanied by meaningful resolution and uncertainty informations. Furthermore, since it does not assume any parameterization of the model space, it enables numerically accurate data sensitivity kernels to be effectively exploited in tomographic inversions. This is a key advantage over discrete tomographic methods, for which data sensitivity kernels are often inaccurate, as they are projected on coarse parameterizations. The most costly task of parameter-free SOLA tomography is the calculation of $\sim N^{2} / 2$ volumetric integrals of the form $\int K_{i} K_{i^{\prime}}$, where $\left(K_{i}, K_{i^{\prime}}\right)$ is a pair of data sensitivity kernels and $N$ the total number of data, what could make intractable very large data sets (e.g., $N \gg 10^{5}$ ). Nevertheless, using modest computational facilities, we have successfully inverted a set of 27070 teleseismic, finite-frequency, $S$-wave time residuals, with focus on imaging and appraising shear-wave velocity anomalies lying in the mantle below Southeast Asia, in the $350-1410 \mathrm{~km}$ depth range.

To conclude, parameter-free SOLA tomography is particularly well suited for regional-scale experiments, for which moderate-size data sets are frequently encountered, so that limited computational resources are sufficient - while enabling quantitative appraisals of tomographic features, and to take full advantage of finite-frequency data sensitivity kernels. 


\section{ACKNOWLEDGMENTS}

The author is grateful to Lapo Boschi (editor), Kasra Hosseini and an anonymous reviewer whose constructive comments have helped to improve this paper, and wishes to thank David Al-Attar for stimulating discussions on Backus-Gilbert theory, Sophie Lambotte for reading an earlier version of this manuscript and Franco Zaroli for invaluable help on some program optimization related to B-trees.

\section{REFERENCES}

Aster, R. C., Borchers, B., \& Thurber, C., 2012. Parameter Estimation and Inverse Problems, Elsevier, revised edn.

Backus, G. \& Gilbert, J. F., 1967. Numerical applications of a formalism for geophysical inverse problems, Geophys. J. Roy. Astr. Soc., 13, 247-276.

Backus, G. \& Gilbert, J. F., 1968. The resolving power of gross Earth data, Geophys. J. Roy. Astr. Soc., 16, 169-205.

Backus, G. \& Gilbert, J. F., 1970. Uniqueness in the inversion of inaccurate gross Earth data, Phil. Trans. Roy. Soc. A., 266(1173).

Bayer, R. \& McCreight, E., 1972. Organization and maintenance of large ordered indexes, Acta Informatica, 1(3), 173-189.

Bogiatzis, P., Ishii, M., \& Davis, T., 2016. Towards using direct methods in seismic tomography: computation of the full resolution matrix using high-performance computing and sparse QR factorization, Geophys. J. Int., 205, 830-836.

Boschi, L., Becker, T. W., Soldati, G., \& Dziewonski, A. M., 2006. On the relevance of Born theory in global seismic tomography, Geophys. Res. Lett., 33(L06302, doi:10.1029/2005GL025063).

Charléty, J., Voronin, S., Nolet, G., Loris, I., Simons, F., Sigloch, K., \& Daubechies, I. C., 2013. Global seismic tomography with sparsity constraints: Comparison with smoothing and damping regularization, J. Geophys. Res, 118, 1-13.

Chevrot, S., Martin, R., \& Komatitsch, D., 2012. Optimized discrete wavelet transforms in the cubed sphere with the lifting scheme - implications for global finite-frequency tomography, Geophys. J. Int., 191, 13911402 .

Dahlen, F. A. \& Nolet, G., 2005. Comment on the paper "On sensitivity kernels for wave-equation transmission tomography" by de Hoop and Van der Hilst, Geophys. J. Int., 163, 949-951.

Dahlen, F. A., Hung, S.-H., \& Nolet, G., 2000. Fréchet kernels for finite-frequency traveltimes - I. theory, Geophys. J. Int., 141, 157-174.

Davies, D. R., Goes, S., Davies, J. H., Schuberth, B. S. A., Bunge, H.-P., \& Ritsema, J., 2012. Reconciling dynamic and seismic models of Earth's lower mantle: The dominant role of thermal heterogeneity, Earth Planet. Sci. Lett., 353-354, 253-269. 
Hosseini, K., 2016. Global multiple-frequency seismic tomography using teleseismic and core-diffracted body waves, Ph.D. thesis, University of Munich (LMU).

Hosseini, K. \& Sigloch, K., 2015. Multifrequency measurements of core-diffracted P waves (Pdiff) for global waveform tomography, Geophys. J. Int., 203(1), 506-521.

Hung, S.-H., Dahlen, F. A., \& Nolet, G., 2001. Wavefront-healing: a banana-doughnut perspective, Geophys. J. Int., 146, 289-312.

Hung, S.-H., Shen, Y., \& Chiao, L.-Y., 2004. Imaging seismic velocity structure beneath the Iceland hotspot: a finite-frequency approach, J. Geophys. Res, 109(B08305, doi:10.1029/2003JB002889).

Kennett, B. \& Engdahl, E., 1991. Traveltimes for global earthquake location and phase identification, Geophys. J. Int., 105, 429-465.

Kennett, B. L. N. \& Bunge, H.-P., 2008. Geophysical Continua, Cambridge University Press.

Maceira, M., Larmat, C., Porritt, R. W., Higdon, D. M., Rowe, C. A., \& Allen, R. M., 2015. On the validation of seismic imaging methods: finite-frequency or ray theory?, Geophys. Res. Lett., 42(2), 323-330.

Maguire, R., Ritsema, J., Bonnin, M., van Keken, P., \& Goes, S., 2018. Evaluating the resolution of deep mantle plumes in teleseismic traveltime tomography, J. Geophys. Res., 123(1), 384-400.

Marquering, H., Nolet, G., \& Dahlen, F. A., 1998. Three-dimensional waveform sensitivity kernels, Geophys. J. Int., 132, 521-534.

Menke, W., 1989. Geophysical Data Analysis: Discrete Inverse Theory, Academic Press, San Diego, revised edn.

Mercerat, E., D., Nolet, G., \& Zaroli, C., 2014. Cross-borehole tomography with correlation delay times, GEOPHYSICS, 79(1), R1-R12.

Montelli, R., Nolet, G., Dahlen, F. A., Masters, G., Engdahl, E. R., \& Hung, S.-H., 2004a. Finite-frequency tomography reveals a variety of plumes in the mantle, Science, 303, 338-343.

Montelli, R., Nolet, G., Masters, G., Dahlen, F. A., \& Hung, S.-H., 2004b. Global P and PP traveltime tomography: rays versus waves, Geophys. J. Int., 158, 636-654.

Nolet, G., 1985. Solving or resolving inadequate and noisy tomographic systems, J. Comp. Phys., 61, 463-482.

Nolet, G., 2008. A breviary of seismic tomography, Cambridge University Press, Cambridge, UK.

Nolet, G. \& Montelli, R., 2005. Optimum parameterization of tomographic models, Geophys. J. Int., 161, 365-372.

Nolet, G., Dahlen, F. A., \& Montelli, R., 2005. Traveltimes and amplitudes of seismic waves: a re-assessment, in Seismic Earth: Analysis of broadband seismograms, vol. 157, pp. 37-48, eds Levander, A. \& Nolet, G., AGU Monograph Series.

Nolet, G., Hello, Y., Lee, S. v. d., Bonnieux, S., Ruiz, M. C., Pazmino, N. A., Deschamps, A., Regnier, M. M., Font, Y., Chen, Y. J., \& Simons, F. J., 2019. Imaging the Galápagos mantle plume with an unconventional application of floating seismometers, Scientific Reports, 9(1), 1326.

Obayashi, M., Yoshimitsu, J., Nolet, G., Fukao, Y., Shiobara, H., Sugioka, H., Miyamachi, H., \& Gao, Y., 2013. Finite frequency whole mantle P wave tomography: Improvement of subducted slab images, Geophys. 
Res. Lett., 40(21), 5652-5657.

Paige, C. C. \& Saunders, M., 1982. LSQR: an algorithm for sparse, linear equations and sparse least squares, A.C.M. Trans. Math. Softw., 8, 43-71.

Parker, R. L., 1994. Geophysical Inverse Theory, Princeton University Press, Princeton.

Pijpers, F. P., 1997. Inversions in astronomy and the SOLA method, In: Springer Science+Business Media New York, Inverse Problems in Wave Propagation, G. Chavent et al. (Eds.).

Pijpers, F. P. \& Thompson, M. J., 1992. Faster formulations of the optimally localized averages method for helioseismic inversions, Astron. Astrophys., 262(L33-L36).

Pijpers, F. P. \& Thompson, M. J., 1994. The SOLA method for helioseismic inversion, Astron. Astrophys., 281, 231-240.

Rawlinson, N., Pozgay, S., \& Fishwick, S., 2010. Seismic tomography: a window into deep Earth, Phys. Earth Planet. Inter, 178(3-4), 101-135.

Rawlinson, N., Fichtner, A., Sambridge, M., \& Young, M. K., 2014. Seismic tomography and the assessment of uncertainty, Adv. Geophys., 55, 1-76.

Ritsema, J., McNamara, A. K., \& Bull, A. L., 2007. Tomographic filtering of geodynamic models: Implications for model interpretation and large-scale mantle structure, J. Geophys. Res, 112(B01303).

Ritsema, J., Deuss, A., van Heijst, H.-J., \& Woodhouse, J. H., 2011. S40RTS: a degree-40 shear-velocity model for the mantle from new Rayleigh wave dispersion, teleseismic traveltime and normal-mode splitting function measurements, Geophys. J. Int., 184, 1223-1236.

Romanowicz, B., 2008. Using seismic waves to image Earth's internal structure, Nature, 451, 266-268.

Ronchi, C., Iacono, R., \& Paolucci, P., 1996. The cubed sphere: a new method for the solution of partial differential equations in spherical geometry, J. Comp. Phys., 124, 93-114.

Sambridge, M., Braun, J., \& McQueen, H., 1995. Geophysical parameterisation and interpolation of irregular data using natural neighbours, Geophys. J. Int., 122, 837-857.

Schuberth, B. S. A., Bunge, H.-P., \& Ritsema, J., 2009. Tomographic filtering of high-resolution mantle circulation models: Can seismic heterogeneity be explained by temperature alone?, Geochem. Geophys. Geosyst., 10(5)(Q05W03), doi:10.1029/2009GC002401.

Schuberth, B. S. A., Zaroli, C., \& Nolet, G., 2015. Traveltime dispersion in an isotropic elastic mantle: strong lower mantle signal in differential-frequency residuals, Geophys. J. Int., 203(3), 2099-2118.

Sigloch, K. \& Nolet, G., 2006. Measuring finite-frequency body-wave amplitudes and traveltimes, Geophys. J. Int., 167, 271-287.

Trampert, J., 1998. Global seismic tomography: the inverse problem and beyond, Inverse Problems, 14, 371385.

Tromp, J., Tape, C., \& Liu, Q., 2005. Seismic tomography, adjoint methods, time reversal and banana-doughnut kernels, Geophys. J. Int., 160, 195-216.

Van der Hilst, R. D. \& de Hoop, M. V., 2005. Banana-doughnut kernels and mantle tomography, Geophys. J. Int., 163, 956-961. 
Voronin, S. \& Zaroli, C., 2018. Survey of computational methods for inverse problems, Recent Trends in Computational Science and Engineering, (http://dx.doi.org/10.5772/intechopen.73332).

Yang, T., Shen, Y., van der Lee, S., Solomon, S., \& Hung, S.-H., 2006. Upper mantle beneath the Azores hotspot from finite-frequency seismic tomography, Earth Planet. Sci. Lett., 250, 11-26.

Zaroli, C., 2010. Global multiple-frequency S-wave tomography of the Earth's mantle, Phd thesis, Strasbourg University.

Zaroli, C., 2016. Global seismic tomography using Backus-Gilbert inversion, Geophys. J. Int., 207(2), 876888.

Zaroli, C., Debayle, E., \& Sambridge, M., 2010. Frequency-dependent effects on global S-wave traveltimes: wavefront-healing, scattering and attenuation, Geophys. J. Int., 182, 1025-1042.

Zaroli, C., Sambridge, M., Lévêque, J.-J., Debayle, E., \& Nolet, G., 2013. An objective rationale for the choice of regularisation parameter with application to global multiple-frequency S-wave tomography, Solid Earth, 4, 357-371.

Zaroli, C., Lambotte, S., \& Lévêque, J.-J., 2015. Joint inversion of normal-mode and finite-frequency S-wave data using an irregular tomographic grid, Geophys. J. Int., 203(3), 1665-1681.

Zaroli, C., Koelemeijer, P., \& Lambotte, S., 2017. Toward seeing the Earth's interior through unbiased tomographic lenses, Geophys. Res. Lett., 44, 11399-11408.

Zhou, Y., Dahlen, F., Nolet, G., \& Laske, G., 2005. Finite-frequency effects in global surface wave tomography, Geophys. J. Int., 182, 1025-1042. 

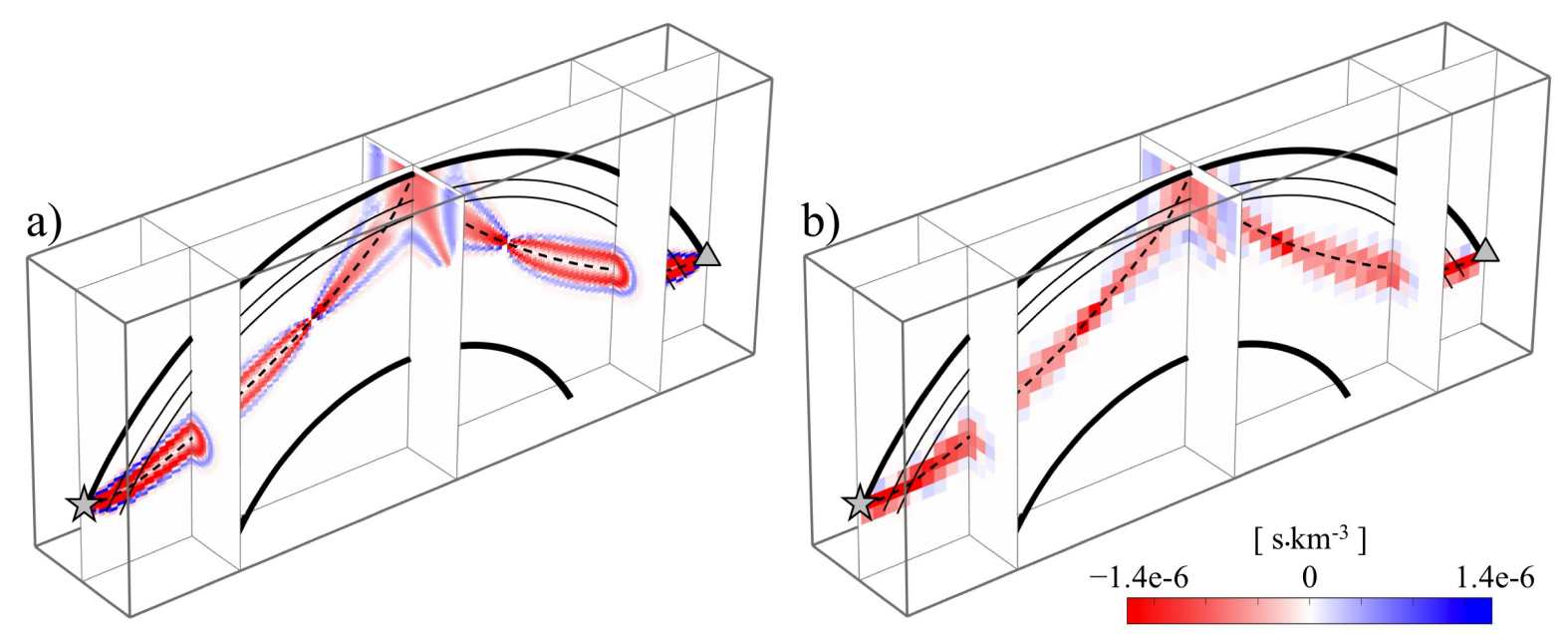

Figure 1. a) Three-dimensional, finite-frequency, SS-phase time-residual sensitivity kernel $\left(120^{\circ}\right.$ epicentral distance, $22 \mathrm{~s}$ central period of a passband Gaussian filter). It is discretized on a local cartesian grid made of regularly spaced $50 \mathrm{~km}$ edge-length cubic cells, spanning a rectangular parallelepiped region (thick gray solid line). The Earth's surface and core-mantle boundary (transition zone) are depicted with thick (thin) black solid lines, respectively, and the earthquake and receiver with a star and triangle. b) Same SS sensitivity kernel but after projection on a tomographic grid that consists in regularly spaced $200 \mathrm{~km}$ edge-length cubic cells; note that the projected sensitivity kernel looks like a 'fat' ray-theoretical kernel - i.e., almost no sensitivity variation all around the geometrical ray path (black dashed line). 

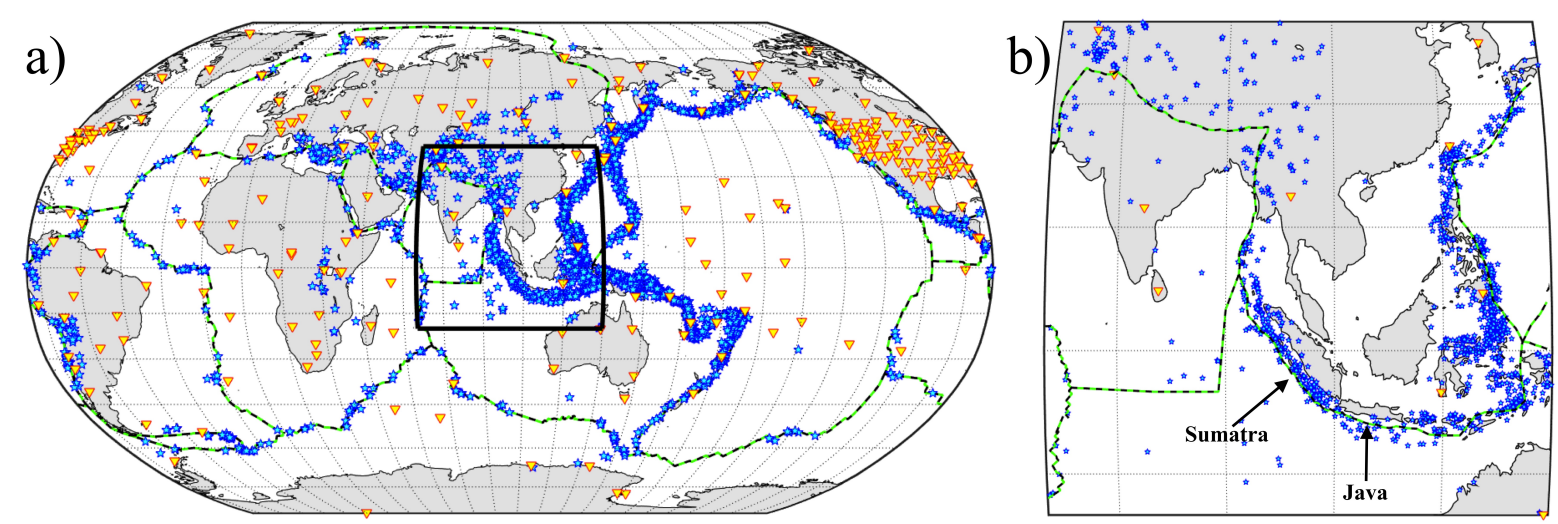

Figure 2. a) Globally distributed earthquakes ( $\sim 4000$ stars $)$ and receivers ( $\sim 250$ triangles) corresponding to the set of teleseismic $S$-wave data used in this study. Tectonic plates are drawn in black-green dashed lines. b) Zoom-in on the 'Southeast Asia' region (black frame). 


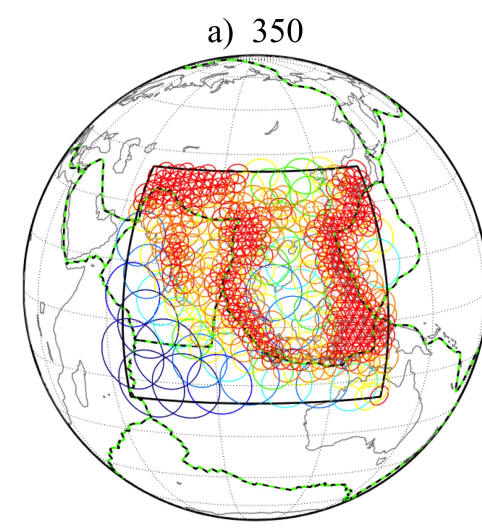

e) 885 b) 465

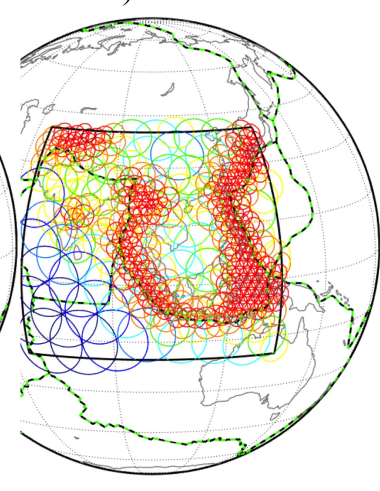

f) 1035 c) 595



d) 735

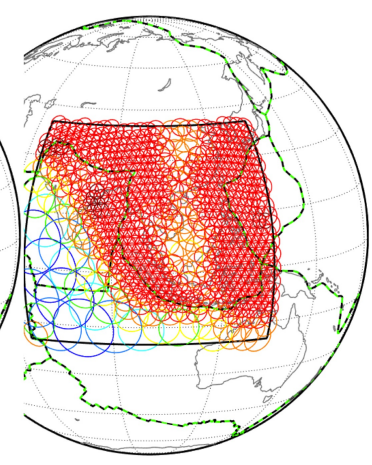

h) $1410[\mathrm{~km}]$

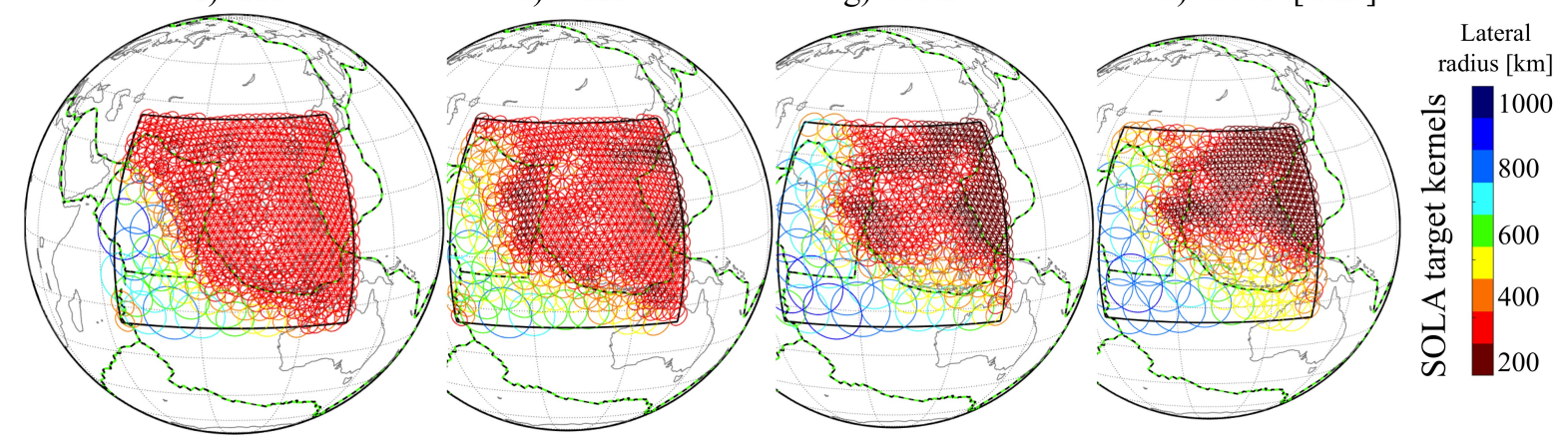

Figure 3. Drawn circles represent lateral, 2-D views of all the 4310 parameter-free SOLA target kernels spanning Southeast Asia at eight different depths in the mantle $(350$ to $1410 \mathrm{~km})$. Note that a query point lies at the centre of each circle, whose the radius is color coded and ray-density driven (see Sect. 2.4). 

a) 350
b) 465
c) 595
d) 735



e) 885

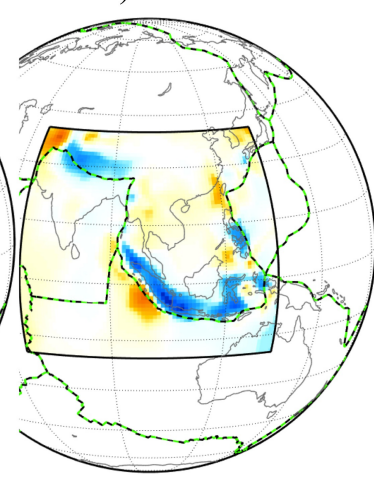

f) 1035
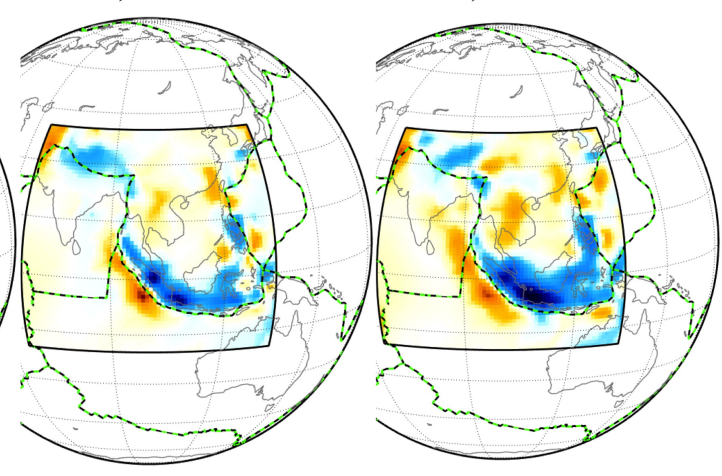

h) $1410[\mathrm{~km}]$

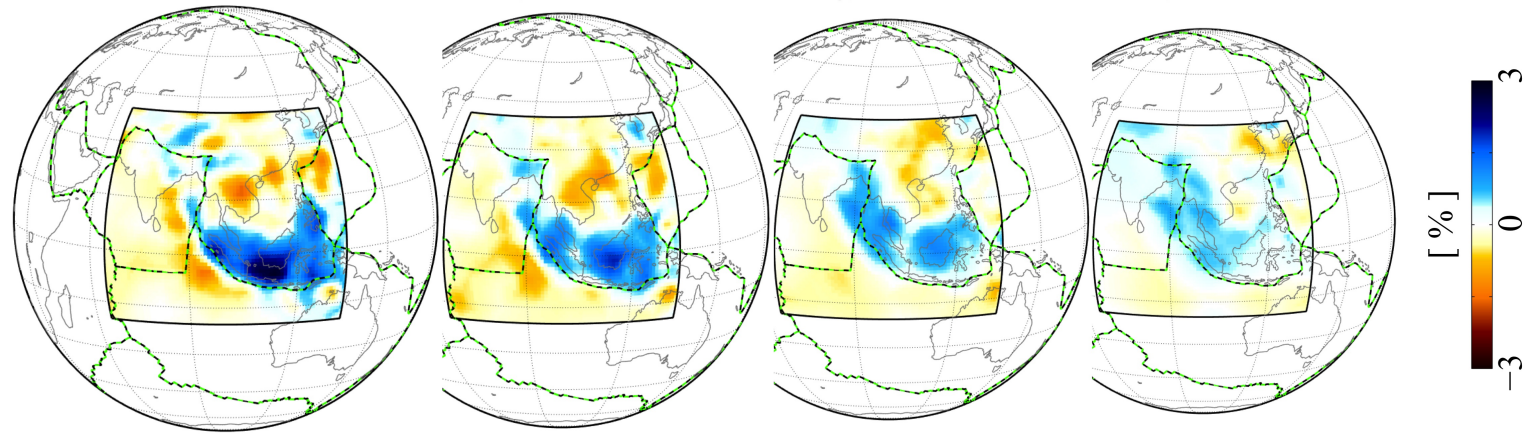

Figure 4. Parameter-free SOLA tomographic images (see Sect. 2.4). 


\section{Christophe Zaroli}

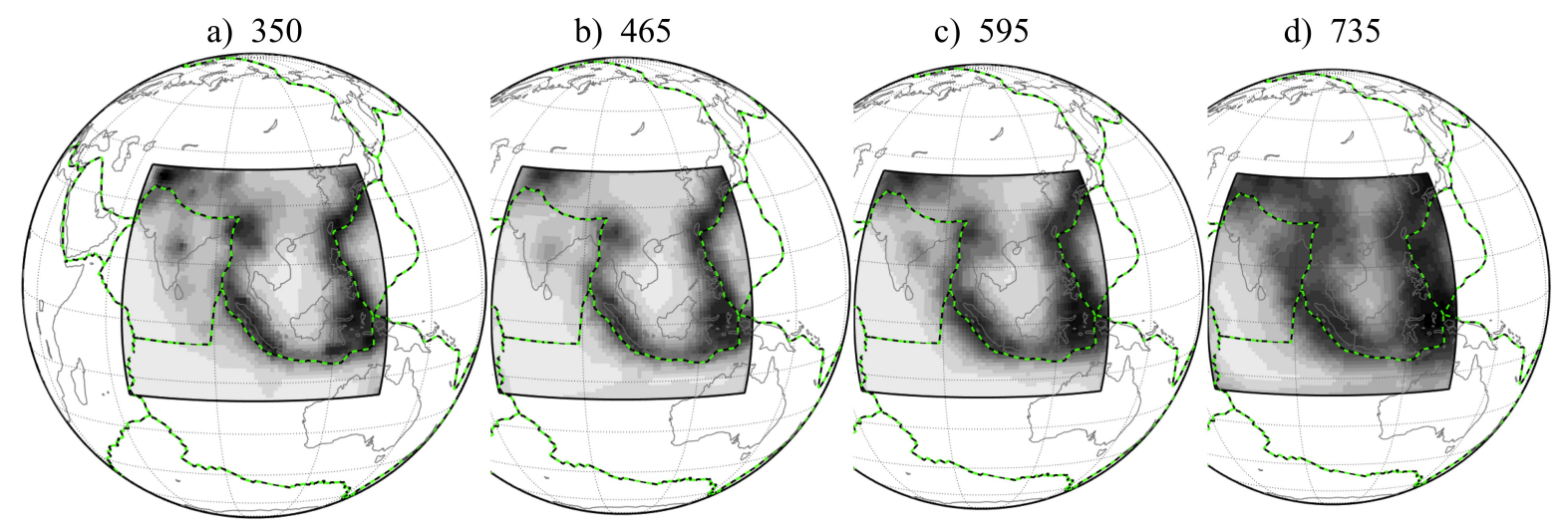

e) 885

f) 1035

g) 1210

h) $1410[\mathrm{~km}]$



Figure 5. Parameter-free SOLA uncertainties (see Sect. 2.4). 

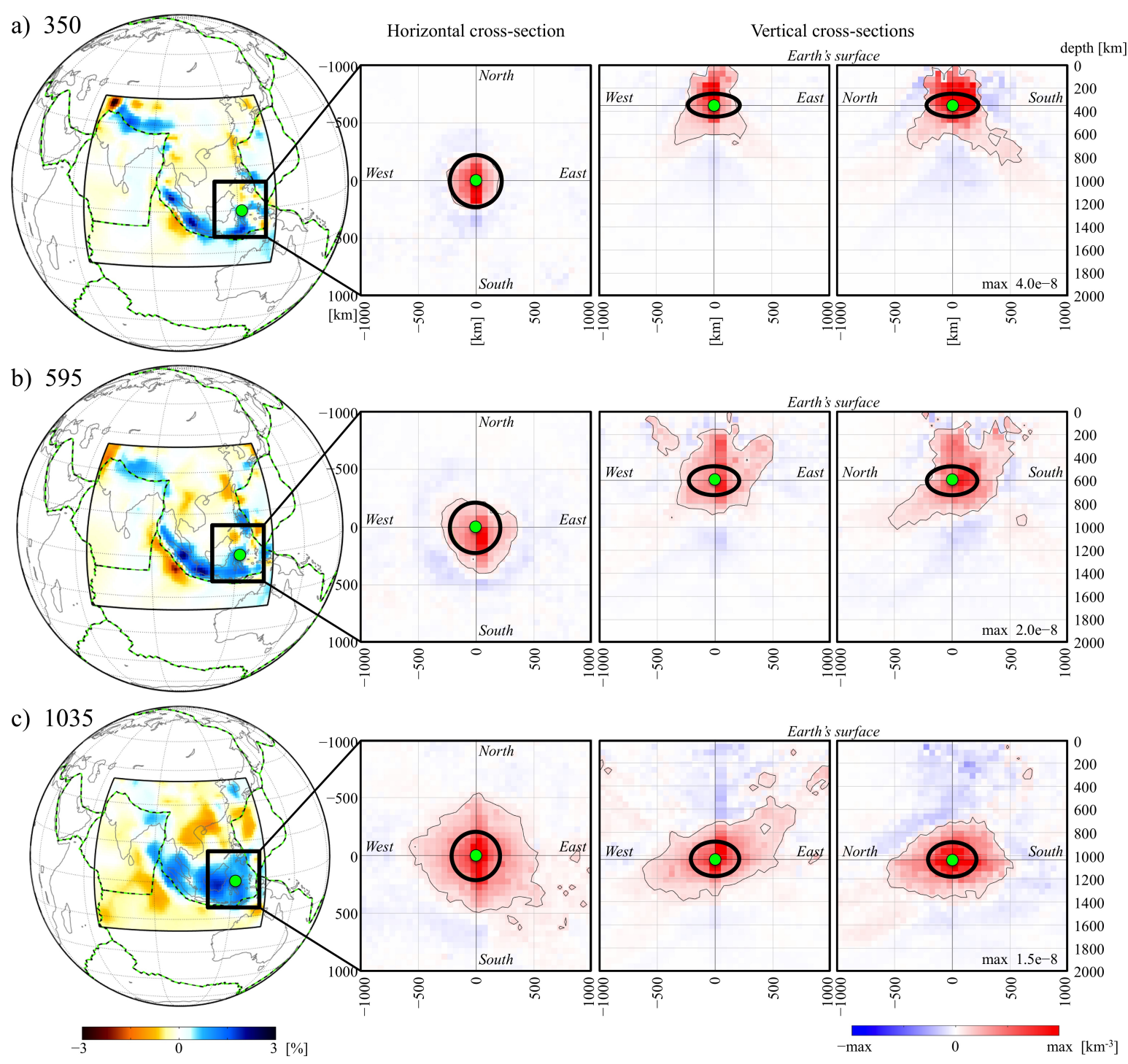

Figure 6. Visualisation of horizontal and vertical cross-sections across three parameter-free SOLA resolving kernels (see Sect. 2.4). The associated three query points are highlighted with green dots; they are located at three selected depths $(350,595$ and $1035 \mathrm{~km})$ below the Sulawesi island. Tomographic images are also displayed. Each drawn black circle (ellipse) represents the horizontal (vertical) spatial extent of the corresponding spheroidshape target kernel, respectively. 


\section{APPENDIX A: COMPUTATIONAL ASPECTS}

We show how to reduce the computational costs and storage requirements involved in setting up and solving the parameter-free SOLA systems (see Sect. 2.2), tailored to teleseismic body-wave mantle tomography within a finite-frequency physical framework.

\section{A1 Sensitivity kernel discretization}

Each finite-frequency data sensitivity kernel $K_{i}$ is discretized on a local cartesian grid that consists in regularly spaced, $50 \mathrm{~km}$ edge-length, cubic cells spanning a rectangular parallelepiped region surrounding $K_{i}$ as illustrated in Fig. 1(a). This avoids using a global grid spanning the whole mantle, which would lead to consider and store much more cells, i.e., several millions versus a few hundreds thousands (see Sect. 2.1), with most cells zero-valued. It is straightforward to transform these local cartesian coordinates to the global cartesian coordinates (Zaroli 2010), which is useful, for example, when evaluating whether two sensitivity kernels $K_{i}$ and $K_{i^{\prime}}$ are simultaneously non-zero valued at the same location (see Sect. A2).

\section{A2 Parameter-free SOLA systems}

In this study, each sensitivity kernel $K_{i}$ is stored in a self-balancing B-tree structure (Bayer \& McCreight 1972). Stored informations are non-zero kernel values $K_{i}(x, y, z)$ and associated local cartesian coordinates $(x, y, z)$. We report that this B-tree approach enables us to efficiently, and elegantly, numerically compute all the integrals encountered in our parameter-free SOLA tomographic problem (see Sect. 2.2), that is: $\int T^{(k)} K_{i}, \int K_{i}, \int K_{i}^{2}, \int K_{i} K_{i^{\prime}}$.

Computing the symmetric matrix $\mathbf{F}^{(\eta)}$ is by far the most costly task in parameter-free SOLA tomography (see Sect. 2.3, App. A6). Thus, we aim to explain how are evaluated all the integrals of the form $\int K_{i} K_{i^{\prime}=i+1} \cdots N$, where $i$ is fixed. These $(N-i)$ integrals correspond to all the elements of the $i$-th row of the upper-right half part of $\mathbf{F}^{(\eta)}$. We proceed as follows: 1) All the non-zero values of $K_{i}$ and their locations (with respect to the local cartesian grid, tailored to $\left.K_{i}\right)$, i.e., $\left\{K_{i}(x, y, z), x, y, z\right\}$, are computed and stored in a B-tree structure referred as $\left.B t\left[K_{i}\right] ; 2\right)$ All the non-zero values of $K_{i^{\prime}}$ and their locations, i.e., $\left\{K_{i^{\prime}}\left(x^{\prime}, y^{\prime}, z^{\prime}\right), x^{\prime}, y^{\prime}, z^{\prime}\right\}$, are computed on the fly; 3) Each triplet $\left(x^{\prime}, y^{\prime}, z^{\prime}\right)$ is searched for in $B t\left[K_{i}\right]$; if it is found, i.e., if there is a triplet $(x, y, z)$ such that $(x, y, z) \equiv\left(x^{\prime}, y^{\prime}, z^{\prime}\right)$ (meaning that $(x, y, z)$ and $\left(x^{\prime}, y^{\prime}, z^{\prime}\right)$ stand for the same global cartesian coordinates, and that both $K_{i}(x, y, z)$ and $K_{i^{\prime}}\left(x^{\prime}, y^{\prime}, z^{\prime}\right)$ are non-zero), then the integral value is updated: $\int K_{i} K_{i^{\prime}} \leftarrow \int K_{i} K_{i^{\prime}}+$ $K_{i}(x, y, z) K_{i^{\prime}}\left(x^{\prime}, y^{\prime}, z^{\prime}\right) \Delta V$, where $\Delta V=50^{3} \mathrm{~km}^{3}$; 4) Repeat 2) and 3) with $i^{\prime}=i+1 \cdots N$.

A few additional remarks. The search time of $\left(x^{\prime}, y^{\prime}, z^{\prime}\right)$ in $B t\left[K_{i}\right]$ is independent of whether 
$\left(x^{\prime}, y^{\prime}, z^{\prime}\right)$ is found or not. This nice property is due to the self-balancing structure itself of B-trees. Since there is at most $B t\left[K_{i}\right]$ and $K_{i^{\prime}}$ to be stored at the time, our approach is not costly in terms of memory footprint. We choose to separate the calculation of all the rows of the upper-right half part of $\mathbf{F}^{(\eta)}$ into parallel tasks, such that the first $N_{1}$ contiguous rows are computed on a first processor, the next $N_{2}$ rows are computed on a second processor, etc. When using the previous algorithm to compute the first $N_{1}$ rows, one actually has to compute once $K_{1}$, twice $K_{2}$, three times $K_{3}$, etc. Therefore, to further speed-up the calculation of the first $N_{1}$ rows (and so on) of the upper-right half part of $\mathbf{F}^{(\eta)}$, all the sensitivity kernels $K_{i}$ are $i$-index sorted such that their computational costs (or a proxy for it, e.g., the total ray path distance) are in decreasing order. The numbers of contiguous rows $\left(N_{1}, N_{2}\right.$, etc) may be chosen such that the work load on every processors is almost identical. Finally, note that new data sets can be easily embedded in existing matrix $\mathbf{F}^{(\eta)}$ and vectors $\mathbf{u}^{(k)}$.

\section{A3 Non-crossing sensitivity kernels}

To alleviate the computational burden of building the matrix $\mathbf{F}^{(\eta)}$, one may try to reduce the number of integrals $\int K_{i} K_{i^{\prime}}$ to be effectively calculated. That is, to identify a priori some pairs of bodywave sensitivity kernels $\left(K_{i}, K_{i^{\prime}}\right)$ that do not cross each other; in which case $\int K_{i} K_{i^{\prime}}=0$. Different strategies could be designed for that purpose. For example, let assume that each sensitivity kernel spans a planar region within the mantle, geometrically defined by the earthquake-receiver great-circle arc. Then, from our ability to identify non-crossing great-circle arcs, one could infer at least some pairs of non-crossing sensitivity kernels. We have adapted this geometrical criterion to take into account volumetric finite-frequency sensitivity kernels, by simply considering 'fat' great-circle arcs.

\section{A4 Resolving kernels}

In parameter-free SOLA tomography, each resolving kernel $A^{(k)}$ has to be computed from (7) in a consistent way with respect to the discretization of data sensitivity kernels (see App. A1). Thus, $A^{(k)}$ is calculated on a grid which consists in regularly spaced, $50 \mathrm{~km}$ edge-length, cubic cells spanning a volumetric region surrounding the query point (see Fig. 6). In practice, to speed-up the computation of $A^{(k)}$, we proceed as follows: 1) Storing $A^{(k)}$ itself in a B-tree; 2) Identifying a priori whether a given sensitivity kernel $K_{i}$ will not cross the volumetric region where we aim at calculating $A^{(k)}$, in which case $K_{i}$ would not contribute to $A^{(k)}$. Step 2) is based on a geometrical criterion similar to App. A3. 


\section{A5 Matrix sparsity}

Preserving the sparsity of the matrix $\mathbf{H}^{(\eta)}$ is crucial in terms of storage, efficiency of LSQR solver, memory footprint, etc. From (24) one sees that the sparsity of $\mathbf{H}^{(\eta)}$ can be optimized, by selecting the first-indexed sensitivity kernel $K_{1}$ such that it leads to minimizing the number of non-zero integrals $\int K_{\mu} K_{1}$, that is, maximizing the number of pairs of non-crossing kernels $\left(K_{\mu}, K_{1}\right)$. The kernel $K_{1}$ is found using a brute-force (computationally cheap) search to count the number of crossing kernels for each individual kernel using the geometrical criterion mentioned in App. A3. We report that, in the case of our application (see Sect. 2.4), $\mathbf{H}^{(\eta)}$ is $\sim 2 \%$ dense.

\section{A6 Computational cost}

Concerning our experiment (see Sect.2.4), for which the number of data is $N=27070$, it takes $\sim 1 \mathrm{wk}$ (CPU time) to compute in parallel using 70 processors (Intel Xeon E5-4657L 2.40 GHz) all the $\sim N^{2} / 2$ elements of the symmetric matrix $\mathbf{F}^{(\eta)}$ - by far the most costly task faced in parameter-free SOLA tomography.

This paper has been produced using the Blackwell Scientific Publications GJI LATEX2e class file. 